\title{
¿La Justicia en Selección Predice las Intenciones de los Candidatos?
}

\section{Does Justice in Selection Predict Candidate's Intentions?}

\author{
Amparo Osca y Blanca López-Araújo \\ UNED
}

\begin{abstract}
Resumen. El estudio analiza las relaciones entre la justicia en selección de personal y las intenciones respecto a la empresa/organización. Para ello se consultó a 261 candidatos reales después de conocer los resultados de un proceso de selección. Se analiza la justicia en los procedimientos, las interacciones y la información así como las intenciones de recomendar la organización, de adquirir sus productos y de iniciar acciones legales. En línea con lo hipotetizado, el resultado de la selección influye en las percepciones de justicia, en las intenciones respecto a la organización y en la intención de adquirir sus productos. Los resultados de las ecuaciones de regresión jerárquica muestran diferencias entre hombres y mujeres. A las mujeres les influye sobre todo la justicia en los procedimientos y a los hombres la justicia en las interacciones y en la información. Se discuten las implicaciones de los resultados para la investigación y la práctica profesional.

Palabras clave: justicia, selección; intenciones respecto a la organización; candidatos, género.
\end{abstract}

\begin{abstract}
The study examines the relationship between justice in personnel selection and the intentions regarding the organization. A total of 261 applicants were surveyed after the selection results were known. A questionnaire was used to measure procedural, interactional and informational justice as well as the intention to recommend the organization, the intention to purchase its products and the intention to pursue legal action. According with ours hypothesis the results of the selection influences in perceptions of justice, in intentions to recommend the organization to others and intentions to purchase its products. Hierarchical regression models calculated to test the hypothesized effects, indicated differences between men and women. For women procedural justice predicted intention towards the organization and for men the significant predictors are interactional justice and informational justice. We discuss the implications of the findings for research and professional practice.

Key words: justice, selection; intentions toward organization; candidates, gender.
\end{abstract}

Desde los años 70 en USA y UK, las empresas y organizaciones cuidan sus prácticas de reclutamiento y selección para tener argumentos con los que defenderse ante posibles demandas por no cumplir la legislación antidiscriminación. Esto ha llevado a desarrollar sistemas de evaluación estructurados, centrados en el contenido de las pruebas y en la consistencia al administrarlas (Harris, 2000). En nuestro país no hay tradición al respecto, sin embargo, la reciente Ley Orgánica 3/2007 para la Igualdad Efectiva de Mujeres y Hombres establece un marco para que, aquellas personas que se sientan discriminadas, puedan ejercer sus derechos y defenderse. Desde esta perspectiva, este artículo tiene un doble objetivo: primero, estudiar cómo influyen las percepciones de justicia en selección sobre las intenciones de los candidatos respecto a la organización que hace la oferta (recomendarla a otros candidatos, adquirir sus productos o iniciar acciones legales en su contra) y, segundo, identificar la influen-

Este trabajo parte del proyecto Percepción de discriminación en los procesos de selección y evaluación del personal (103-06) que ha sido subvencionado por el Instituto de la Mujer. La correspondencia sobre este artículo deberá dirigirse a Amparo Osca, Facultad de Psicología, UNED, c/ Juan del Rosal, 10, 28040 Madrid. E-mail: aosca@psi. uned.es cia en esas relaciones del resultado de la selección (ser o no ser seleccionado) y del género de los candidatos.

Ya en los años 90, Gilliland (1993) y Smither, Reilly, Millsap y Pearlman (1993) alertaron de que los procedimientos que no se consideraban razonables en general, y no relacionados con el puesto en particular, generaban entre los candidatos rechazo y actitudes negativas hacia la organización, lo que incluso podría llevarles a iniciar acciones legales. Saks, Leck y Saunders (1995) analizaron con un grupo de estudiantes si la introducción de cuestiones discriminatorias en un formulario de admisión influía en sus intenciones respecto a la organización. Según sus resultados los sujetos que completaban cuestionarios no discriminatorios tenían reacciones más positivas sobre el atractivo de la organización, la intención de aceptar el trabajo y la posibilidad de recomendar la organización. También con candidatos reales se encontró que las reacciones a las pruebas se relacionaban con las intenciones de recomendar a la organización (Smither et al., 1993), sin embargo,el uso de formularios con preguntas no apropiadas (respecto al sexo, la edad, etc.) influía en las intenciones de litigar contra la organización (Wallace, Page y Lippstreu, 2006).

Respecto a la justicia de las pruebas, Gilliland y Chan (2001) plantearon que la falta de justicia influye 
en las intenciones de demandar a la organización, como encuentran otros autores (Goldman, 2001; Steiner y Gilliland, 2001). Bauer y su equipo encontraron que la justicia procedimental influía en el atractivo de la organización y en las intenciones hacia ésta (Bauer, Maertz, Dolen y Campion, 1998) y también en las intenciones de recomendarla (Bauer y cols., 2001). Años después, Holtz, Ployhart y Domínguez (2005) observaron igualmente que la justicia en selección (concretamente en la relación con el puesto y en el proceso) se asociaba de forma importante con el atractivo y con la intención de recomendar a la organización. En vista de lo anterior, formulamos la siguiente hipótesis:

Hipótesis 1: Se esperan relaciones entre la percepción de justicia en selección y las intenciones de los candidatos respecto a la organización que hace la oferta, de forma que aquellos que perciban más justicia, mostrarán intenciones más favorables (concretamente, mayor intención de recomendar a la organización, de adquirir sus productos y menor de iniciar acciones legales en su contra).

No obstante, se ha señalado que uno de los aspectos que más influye en la percepción de lo que ocurre durante la selección es el resultado, pues los candidatos seleccionados tienen, en general, percepciones más positivas (p. ej. Elkins y Phillips, 2000) y, al contrario, los que son rechazados tienden a considerar los procesos más injustos reduciendo el riesgo sobre su autoestima (Chan, Schmitt, Sacco y Deshon, 1998). El meta-análisis de Hausknecht, Day y Thomas (2004) también puso de manifiesto la influencia del resultado sobre las percepciones en selección. Recientemente en un estudio experimental, Rolland y Steiner (2007) encuentran que el resultado se relaciona con tres dimensiones de la justicia procedimental consideradas y con las intenciones de recomendar. Gilliland (1994) anticipaba más reacciones negativas si los resultados eran más bajos que los esperados y los procedimientos utilizados se consideraban injustos. Es decir, ser rechazado, si se han utilizado procedimientos adecuados, tiene un impacto más negativo en los candidatos, reafirmando la idea del sesgo y las posibles interacciones. En esta línea, Ployhart y Ryan (1997) obtuvieron interacciones entre la percepción de justicia y el resultado sobre las intenciones de recomendar, de forma que los procesos justos están relacionados con intenciones más favorables y, sobre todo, en aquellos sujetos con mejores resultados. Así la justicia interacciona con el resultado de manera que cuándo el resultado es favorable la justicia apenas tiene efecto (Brockner y Wiesenfeld, 2005), sin embargo, con un resultado negativo, la justicia juega un papel clave, mejorando la percepción de justicia (Cropanzano y Greenberg, 1997). En el estudio de Bauer y cols. (1998) sobre la influencia de la justicia procedimental en el atractivo y las intenciones hacia la organización, se observó que el peso de la justicia disminuye cuando se considera el resultado. Sin embargo, más tarde Bauer y cols. (2001) en una simulación con estudiantes, constataron que la justicia procedimental predecía las intenciones de recomendar, incluso después de controlar los resultados (en una prueba de capacidad cognitiva). En el estudio de Maertz, Bauer, Mosley, Posthuma y Campion (2004), las percepciones de justicia procedimental tenían una escasa influencia en las intenciones hacia la organización (después de controlar las puntuaciones iniciales en esta variable), pero incluso esta influencia disminuía al introducir en los análisis los resultados de la selección. Sin embargo, Anderson y Goltsi (2006), encontraron que también los candidatos aceptados pueden mostrar efectos negativos por la percepción de falta de justicia (procedimental o distributiva). Basándonos en todo lo anterior, formulamos nuestra segunda hipótesis:

Hipótesis 2: Se esperan interacciones entre las percepciones de justicia y el resultado de la selección sobre las intenciones hacia la organización de manera que el efecto de un resultado negativo podría compensarse con una elevada percepción de justicia.

Respecto a las diferencias de género, algunos trabajos muestran que hombres y mujeres responden de forma diferente a las percepciones de justicia. Por ejemplo, Anderson y Shinew (2003) constatan diferencias significativas en tres escalas de justicia organizacional, procedimental, distributiva e interaccional. No obstante, cuándo se evalúa la justicia relacionada con los procesos de selección, la investigación no encontró grandes diferencias en función del género. Esto llama la atención si se tienen en cuenta los peores resultados de las mujeres en el mundo del trabajo en general, y en sus ingresos y posibilidades de promoción, en particular (Instituto de la Mujer, 2008). Además, es posible que dadas las diferencias en los roles sociales asignados, las mujeres prefieran un trato más cercano y que se les deje participar y los hombres relaciones más jerárquicas (Rosener, 1990). En nuestro contexto, en el estudio Osca y García-Salmones (2004), las mujeres sólo presentaron puntuaciones estadísticamente menores que los hombres en dos de las dimensiones de justicia procedimental evaluadas, información sobre el puesto de trabajo y posibilidad de demostrar los conocimientos y habilidades durante la selección. En la misma dirección, Viswesvaran y Ones (2004) sólo observaron diferencias significativas entre hombres y mujeres en una de las veintidós dimensiones de la justicia evaluadas consistencia en la administración de las pruebas de forma que los hombres puntúan más alto que las mujeres. No obstante, es probable que el género no influya de forma directa pero puede que lo haga a través de su interacción con terceras variables. Así Bernerth (2005), basándose en la Teoría de la congruencia de rol de 
Eagly y Karau (2002), analizó las diferencias de género en los resultados de la selección. Según este planteamiento los hombres, siguiendo la dimensión agente propia de su rol de género, estarían menos dispuestos a aceptar aquella información que no concuerde con su imagen personal y las mujeres, cuyo rol les lleva a ser más emotivas, serían más susceptibles de evaluaciones externas. Sus análisis apoyan sus hipótesis ya que las mujeres percibían menos justicia que los hombres cuando el resultado de la selección no era favorable y más si era favorable. Por ello, planteamos las dos hipótesis siguientes:

Hipótesis 3: No se esperan diferencias significativas en la percepción de justicia en selección entre hombres y mujeres.

Hipótesis 4: Se esperan diferencias en cómo hombres y mujeres reaccionan a lo sucedido durante la selección, de forma que las dimensiones de justicia que más influirán sobre las intenciones serán diferentes para unos y otras.

\section{Método}

\section{Procedimiento}

La muestra procede de todo el estado español y se obtuvo a través de contactos personales de los miembros del equipo de investigación y de alumnos de Doctorado de la UNED. La única condición fue haber participado recientemente en un proceso de selección y que el proceso incluyera diferentes fases e instrumentos y, específicamente, una entrevista. Antes de comenzar se explicaron los objetivos del estudio y se garantizó la confidencialidad de la información aportada.

\section{Participantes}

La muestra estaba compuesta por 261 candidatos, el $50.2 \%$ eran hombres (131). La media de edad era 30.89 años (D.T. $=8.5)$ para los hombres y 29.44 $(D . T=6.5)$ para las mujeres. El nivel de estudios era bastante elevado: Universitarios $38.5 \%$ y $41.4 \%$, Estudios medios $21.5 \%$ y $26.6 \%$, FP $15.4 \%$ y $14.9 \%$, Bachillerato $15.4 \%$ y $13.3 \%$ y Sin estudios $9.2 \%$ y $3.9 \%$, para hombres y mujeres respectivamente. Las mujeres eran algo más jóvenes que los hombres $(F=2.37, p>0.05)$ y tenían un mayor nivel formativo $(F=2.25, p>0.05)$ aunque, las diferencias no alcanzaron el nivel de significación mínimo. En el proceso de selección en el que participaron fueron seleccionados un $72.3 \%$ de las mujeres y un $64.3 \%$ de los hombres. No hubo diferencias significativas entre seleccionados y no seleccionados ni en edad $(F=2.45, p>0.05)$ ni en nivel formativo $(F=2.16, p>0.05)$.

\section{Instrumentos}

La justicia en selección se midió con tres de las subescalas de la Escala de Justicia Organizacional de Colquitt (2001) pero referidas a situaciones de selección de personal. Para determinar si la estructura factorial de la escala coincidía con la original se hizo un análisis factorial exploratorio con extracción de componentes principales (Rotación Varimax) y un análisis del contenido de los ítems. Así, se distinguieron tres subescalas que coincidían con las de Colquitt: justicia en los procedimientos o procedimental con 9 ítems (p. ej. "Cree que ha podido expresar sus puntos de vista y sentimientos durante el proceso de selección”), justicia en las relaciones o interaccional con 4 ítems (p. ej. "Cree que le trataron de manera educada") y justicia en la información o informativa con 5 ítems (p. ej. "Cree que le comunicaron los detalles del proceso de forma oportuna”). Estos ítems se evaluaron con escalas de respuesta Likert con siete opciones que oscilaban entre 1 Totalmente en desacuerdo y 7 Totalmente de acuerdo.

Para evaluar las intenciones respecto a la organización que hace la oferta se siguió la propuesta de Macan, Avedon, Paese y Smith (1994) y de Smither y cols. (1993) y se diseñaron tres ítems que preguntaban por tres aspectos: intención de recomendar la organización a otros candidatos (ítem "¿Cuáles son sus intenciones de recomendar esta organización/empresa a otros posibles candidatos?"), de comprar sus productos (ítem “¿Cuáles son sus intenciones de adquirir los productos de esta organización?") y de litigar (ítem "¿Cuáles son sus intenciones de iniciar de acciones legales en contra de la organización/empresa?"). Como en el caso anterior, las preguntas se evaluaron con escalas de respuesta Likert con siete opciones de respuesta (1 Muy bajas y 7 Muy altas).

En la Tabla 1 se presentan las medias, desviaciones típicas, fiabilidades (Alpha de Cronbach) y correlaciones entre variables.

\section{Resultados}

Los resultados de los análisis de varianza para poner a prueba la hipótesis 1 relativa a las diferencias entre seleccionados y no seleccionados (Tabla 2) muestran, en línea con lo esperado, que los candidatos seleccionados presentan puntuaciones significativamente más altas en las tres subescalas de justicia: justicia procedimental $(F=105.23, p<0.001)$, justicia relacional $(F=70.15, p<0.001)$ y justicia en la información $(F=61.96, p<0.001)$. Asimismo, los seleccionados tienen mayores intenciones de recomendar la organización a otros candidatos $(F=71.18, p<0.001)$ y de adquirir sus productos $(F=20.59, p<0.001)$ que los no seleccionados, aunque no aparecen diferencias significativas en la intención de iniciar acciones legales contra la organización $(F=1.68, p>0.05)$. 
Tabla 1. Correlaciones entre variables

\begin{tabular}{|c|c|c|c|c|c|c|c|c|c|}
\hline Variables & $\bar{X}$ & DT & 1 & 2 & 3 & 4 & 5 & 6 & 7 \\
\hline 1. Resultado SP & 1.68 & .47 & - & $.51 * * *$ & $.41 * * *$ & $.41 * * *$ & $.41 * * *$ & $.30 * * *$ & -.12 \\
\hline 2. Justicia Procedimental & 4.60 & 1.00 & $.58 * * *$ & .84 & $.71 * * *$ & $.81 * * *$ & $.64 * * *$ & $.46^{* * *}$ & -.14 \\
\hline 3. Justicia Relacional & 5.58 & .96 & $.51 * * *$ & $.75 * * *$ & .82 & $.69 * * *$ & $.56^{* * *}$ & $.42 * * *$ & $-.22 * *$ \\
\hline 4. Justicia Información & 4.69 & 1.24 & $.50 * * *$ & $.83 * * *$ & $.68 * * *$ & .91 & $.57 * * *$ & $.35^{* * *}$ & $-.15 \dagger$ \\
\hline 5. Recomendar org.anización & 4.57 & 1.51 & $.53 * * *$ & $.60 * * *$ & $.58 * * *$ & $.56 * * *$ & - & $.49 * * *$ & -.02 \\
\hline 6. Adquirir productos & 3.69 & 1.88 & $.28 * * *$ & $.52 * * *$ & $.46^{* * *}$ & $.55^{* * *}$ & $.67 * * *$ & - & .05 \\
\hline 7. Iniciar acciones legales & 1.40 & .89 & -.04 & -.01 & -.13 & -.02 & -.09 & -.02 & - \\
\hline
\end{tabular}

Nota. Resultado en el proceso de SP: 1 No seleccionado y 2 Seleccionado. La fiabilidad de las variables en la diagonal. Correlaciones de hombres por debajo de la diagonal, de mujeres por encima. $\uparrow<.10 * \mathrm{p}<.05 * * \mathrm{p}<.01 * * * \mathrm{p}<.001$

Tabla 2. Diferencias en justicia organizacional entre seleccionados y no seleccionados

\begin{tabular}{|c|c|c|c|c|}
\hline Variable & Resultado & $\bar{X}$ & D.T. & $F$ \\
\hline \multirow[t]{2}{*}{ Justicia Procedimental } & Seleccionados & 4.96 & .79 & \multirow[t]{2}{*}{$105.23 * * *$} \\
\hline & No seleccionados & 3.81 & .94 & \\
\hline \multirow[t]{2}{*}{ Justicia Relacional } & Seleccionados & 5.89 & .83 & \multirow[t]{2}{*}{$70.15^{* * *}$} \\
\hline & No seleccionados & 4.93 & .90 & \\
\hline \multirow[t]{2}{*}{ Justicia Informativa } & Seleccionados & 5.06 & 1.06 & \multirow[t]{2}{*}{$61.96 * *$} \\
\hline & No seleccionados & 3.89 & 1.20 & \\
\hline \multirow[t]{2}{*}{ Recomendar la organización } & Seleccionados & 5.05 & 1.26 & \multirow[t]{2}{*}{$71.18 * * *$} \\
\hline & No seleccionados & 3.54 & 1.48 & \\
\hline \multirow[t]{2}{*}{ Intención adquirir productos } & Seleccionados & 4.03 & 1.90 & \multirow[t]{2}{*}{$20.59 * * *$} \\
\hline & No seleccionados & 2.91 & 1.59 & \\
\hline \multirow[t]{2}{*}{ Inicia acciones legales } & Seleccionados & 1.36 & .91 & \multirow[t]{2}{*}{1.68} \\
\hline & No seleccionados & 1.51 & .85 & \\
\hline
\end{tabular}

${ }^{*} \mathrm{p}<.05 * * \mathrm{p}<.01 * * * \mathrm{p}<.001$

Para poner a prueba la hipótesis relativa al efecto directo del género en la percepción de justicia se han realizado tres análisis de varianza. Como se puede comprobar en la Tabla 3, y confirmando la hipótesis planteada, no aparecen diferencias significativas entre hombres y mujeres en ninguna de las dimensiones de justicia evaluadas: justicia procedimental, relacional e informativa.

Para contrastar las hipótesis 2 relativa a las consecuencias de la justicia y de los resultados de la selección sobre las intenciones, se han realizado tres ecua- ciones de regresión por pasos, introduciendo en el primero las tres dimensiones de justicia y los resultados de la selección y, en el segundo, la interacción entre ambos, según se recomienda para determinar el efecto de modulación (Cohen y Cohen, 1983; Landsbergis, Schnall, Warren, Pickering y Schwartz, 1994; Zedeck, 1971). Además se han realizado análisis independientes para hombres y mujeres con el objetivo de ver si les afectan aspectos distintos. En la Tabla 4 se muestra cómo la intención de recomendar la organización a otros candidatos se predice en los hombres por la jus-

Tabla 3. Diferencias en justicia organizacional entre hombres y mujeres

\begin{tabular}{llrrr}
\hline Variable & Resultado & $\bar{X}$ & D.T. & F \\
\hline Justicia Procedimental & Hombres & 4.61 & .96 & .05 \\
\multirow{2}{*}{ Justicia Relacional } & Mujeres & 4.58 & 1.03 & .95 \\
\multirow{2}{*}{ Justicia Informativa } & Hombres & 5.55 & .98 & .42 \\
& Mujeres & 5.62 & .17 & .62 \\
\hline
\end{tabular}

$* \mathrm{p}<.05 \quad * * \mathrm{p}<.01 \quad * * * \mathrm{p}<.001$ 
Tabla 4. Resultados de las ecuaciones de regresión para predecir la intención de recomendar la organización a otros candidatos

\begin{tabular}{|c|c|c|c|c|}
\hline \multicolumn{2}{|c|}{ Resultados hombres } & \multirow{2}{*}{$\begin{array}{l}\beta \\
.14 \\
.21^{*} \\
.15 \\
.27^{* * *}\end{array}$} & \multirow{2}{*}{$\frac{F}{24.61 * * *}$} & \multirow{2}{*}{$\frac{\Delta R^{2}}{.42 * * *}$} \\
\hline 1. & $\begin{array}{l}\text { - Justicia procedimental } \\
\text { - Justicia relacional } \\
\text { - Justicia información } \\
\text { - Resultado selección }\end{array}$ & & & \\
\hline 2. & $\begin{array}{l}\text { - Justicia procedimental } \\
\text { - Justicia relacional } \\
\text { - Justicia información } \\
\text { - Resultado selección } \\
\text { - Justicia procedimental * Resultado } \\
\text { - Justicia relacional * Resultado } \\
\text { - Justicia información * Resultado }\end{array}$ & $\begin{array}{r}-.64 \\
.53 \\
.35 \\
-.07 \\
1.65 \\
-.73 \\
-.33\end{array}$ & $14.67 * * *$ & .43 \\
\hline & lujeres & $\beta$ & $F$ & $\Delta R^{2}$ \\
\hline 1. & $\begin{array}{l}\text { - Justicia procedimental } \\
\text { - Justicia relacional } \\
\text { - Justicia información } \\
\text { - Resultado selección }\end{array}$ & $\begin{array}{l}.37 * * * \\
.18 \\
.10 \\
.10\end{array}$ & $24.83 * * *$ & $.43 * * *$ \\
\hline 2. & $\begin{array}{l}\text { - Justicia procedimental } \\
\text { - Justicia relacional } \\
\text { - Justicia información } \\
\text { - Resultado selección } \\
\text { - Justicia procedimental * Resultado } \\
\text { - Justicia relacional * Resultado } \\
\text { - Justicia información * Resultado }\end{array}$ & $\begin{array}{r}1.26^{*} \\
-.27 \\
-.10 \\
.24 \\
-1.56 \\
.91 \\
.29\end{array}$ & $12.95 * * *$ & .43 \\
\hline
\end{tabular}

Nota. Resultado en el proceso de SP: 1 No seleccionado y 2 seleccionado.

$\uparrow<.10 * \mathrm{p}<.05 * * \mathrm{p}<.01 * * * \mathrm{p}<.001$

ticia interaccional $(\beta=.21 ; p<0.05) \mathrm{y}$, sobre todo, por el resultado de la selección $(\beta=.27 ; p<0.001)$. Cuando, en el segundo paso de la ecuación de regresión, se introducen las interacciones no se encuentran valores significativos. En el caso de las mujeres la intención de recomendar se predice por la justicia procedimental ( $\beta=.37 ; p<0.001)$, dato que se confirma cuando se introduce en la ecuación el segundo paso $(\beta=1.26 ; p<0.05)$ con las interacciones. Como se puede observar tanto en hombres como en mujeres, los porcentajes de varianza explicada son elevados (42\% y $43 \%$ respectivamente).

Respecto a la intención de adquirir los productos de la organización, la Tabla 5 muestra que para los hombres la variable más importante es la justicia en la información $(\beta=.38 ; p<0.01)$, y de nuevo el resultado de la selección no influye ni directamente, ni a través de su interacción con las dimensiones de justicia. Los análisis para las mujeres señalan que la variable más importante para explicar la intención de adquirir los productos es la justicia procedimental $(\beta=.38 ; p<0.01)$, y el resultado de la selección no influye tampoco, ni de forma directa, ni modulada.

Por ultimo, y respecto a la intención de iniciar acciones legales (Tabla 6), para los hombres la única variable significativa es la justicia interaccional $(\beta=-.26 ; p<0.05)$, de forma que los que perciben más justicia muestran una menor tendencia. De nuevo, para las mujeres los resultados son diferentes, aunque el primer paso de la regresión no arroja valores significativos, sí lo hace el segundo. Como se puede comprobar, son significativas las contribuciones de la justicia en los procedimientos $(\beta=1.53 ; p<0.05)$ y de la justicia en la información $(\beta=-1.86 ; p<0.01) \mathrm{y}$, además, aparecen dos interacciones significativas: la justicia en los procedimientos por los resultados de la selección $(\beta=-2.40 ; p<0.05)$ y la justicia en la información por los resultados de la selección $(\beta=2.64 ; p<0.01)$, aunque no aumentan el porcentaje de varianza explicada por las relaciones directas, consideradas en el paso anterior.

\section{Discusión y conclusiones}

Como se ha señalado en la introducción, este artículo tenía un doble objetivo: analizar cómo influyen las percepciones de justicia en selección sobre las intenciones de los candidatos e identificar, en esas relaciones, el peso del resultado de la selección y del género de los candidatos. Para ello se utilizó una muestra de candidatos reales participantes en procesos de selección, algo destacable si se tiene en cuenta que buena parte de la investigación sobre la justicia se ha realizado con estudiantes y en contextos simulados (p. ej. 
Tabla 5. Resultados de las ecuaciones de regresión para predecir la intención de adquirir los productos de la organización

\begin{tabular}{|c|c|c|c|c|}
\hline \multicolumn{2}{|c|}{ Resultados hombres } & \multirow{2}{*}{$\begin{array}{l}\beta \\
.12 \\
.13 \\
.38^{* *} \\
-.05\end{array}$} & \multirow{2}{*}{$\frac{F}{13.24 * * *}$} & \multirow{2}{*}{$\frac{\Delta R^{2}}{.29 * * *}$} \\
\hline 1. & $\begin{array}{l}\text { - Justicia procedimental } \\
\text { - Justicia relacional } \\
\text { - Justicia información } \\
\text { - Resultado selección }\end{array}$ & & & \\
\hline 2. & $\begin{array}{l}\text { - Justicia procedimental } \\
\text { - Justicia relacional } \\
\text { - Justicia información } \\
\text { - Resultado selección } \\
\text { - Justicia procedimental * Resultado } \\
\text { - Justicia relacional * Resultado } \\
\text { - Justicia información * Resultado }\end{array}$ & $\begin{array}{r}-.85 \\
.62 \\
.74 \\
-.16 \\
2.00 \\
-1.10\end{array}$ & -.65 & .30 \\
\hline & nujeres & $\beta$ & $F$ & $\Delta R^{2}$ \\
\hline 1. & $\begin{array}{l}\text { - Justicia procedimental } \\
\text { - Justicia relacional } \\
\text { - Justicia información } \\
\text { - Resultado selección }\end{array}$ & $\begin{array}{l}.38 * * \\
.20 \\
-.12 \\
.06\end{array}$ & $9.42 * * *$ & $.21 * * *$ \\
\hline 2. & $\begin{array}{l}\text { - Justicia procedimental } \\
\text { - Justicia relacional } \\
\text { - Justicia información } \\
\text { - Resultado selección } \\
\text { - Justicia procedimental } * \text { Resultado } \\
\text { - Justicia relacional * Resultado } \\
\text { - Justicia información } * \text { Resultado }\end{array}$ & $\begin{array}{r}.67 \\
.05 \\
-.68 \\
-.36 \\
-.44\end{array}$ & $5.65 * * *$ & .21 \\
\hline
\end{tabular}

Nota. Resultado en el proceso de SP: 1 No seleccionado y 2 seleccionado.

$\dagger<.10 * \mathrm{p}<.05 \quad * * \mathrm{p}<.01 \quad * * * \mathrm{p}<.001$

Saks y cols., 1995; Wallace y cols., 2006). Además el estudio avanza respecto a trabajos anteriores (p. ej. Bauer y cols., 1998; Maertz y cols., 2004) ya que además de la justicia procedimental se utilizan otras dos dimensiones de la justicia, la interaccional y la justicia en la información.

Como se esperaba, las tres dimensiones de justicia evaluadas, en los procedimientos, en las relaciones y en la información, se relacionan en la dirección esperada con dos de las tres intenciones consideradas (intención de recomendar la organización a otros candidatos e intención de adquirir sus productos), aunque hay diferencias en función del género y de la intención evaluada. Por otra parte, la intención de iniciar acciones legales ofrece resultados que merecen algún comentario adicional. Los descriptivos en esta variable muestran que ni los hombres ni las mujeres de esta investigación han considerado esta posibilidad, probablemente porque en nuestro país no hay tradición de demandas por procedimientos injustos o discriminatorios en selección de personal. Esta puede ser la razón de la ausencia de relaciones claras o con peso predictivo relevante. Recordar que sólo la justicia en las relaciones se vincula con las intenciones de litigar, pero explicando porcentajes de varianza bajos (entre un 2 y un 5\%).

También en línea con la hipótesis y la bibliografía examinada, los resultados de la selección (ser o no ser contratado) influyen tanto en las percepciones de justi- cia como en las intenciones de recomendar y de adquirir los productos de la organización. Sin embargo, cuando en los análisis se introducen, además de los resultados, las percepciones de justicia, el peso de los resultados se anula, en contra de lo hallado en otros trabajos (Bauer y cols., 1998; Maertz y cols., 2004). Como excepción, ser contratado (junto a la percepción de justicia relacional) sólo influye a los hombres y en su intención de recomendar la organización a otros candidatos.

Confirmando la tercera hipótesis y en línea con la investigación sobre el tema (Osca y García-Salmones, 2004; Viswesvaran y Ones, 2004), no se encuentran diferencias en las percepciones que hombres y mujeres tienen de lo que ocurre durante la selección, ni en los procedimientos utilizados, ni en las relaciones con los seleccionadores, ni en la información recibida sobre el proceso. No obstante, como en otros trabajos, se confirma que les afectan aspectos diferentes de la justicia (p. ej. Moliner, Martínez-Tur, Peiró y Ramos, 2005; Sweeney y McFarlin, 1997). Así, la intención de recomendar la organización se explica en los hombres por la justicia en las relaciones y en las mujeres, por la justicia en los procedimientos. Por su parte la intención de adquirir sus productos, se predice en los hombres por la justicia en la información y en las mujeres, de nuevo por la justicia en los procedimientos, explicando en ambos casos, porcentajes de varianza muy importantes. Por último, y como se ha avanzado, la intención de 
Tabla 6. Resultados de las ecuaciones de regresión para predecir la intención de iniciar acciones legales

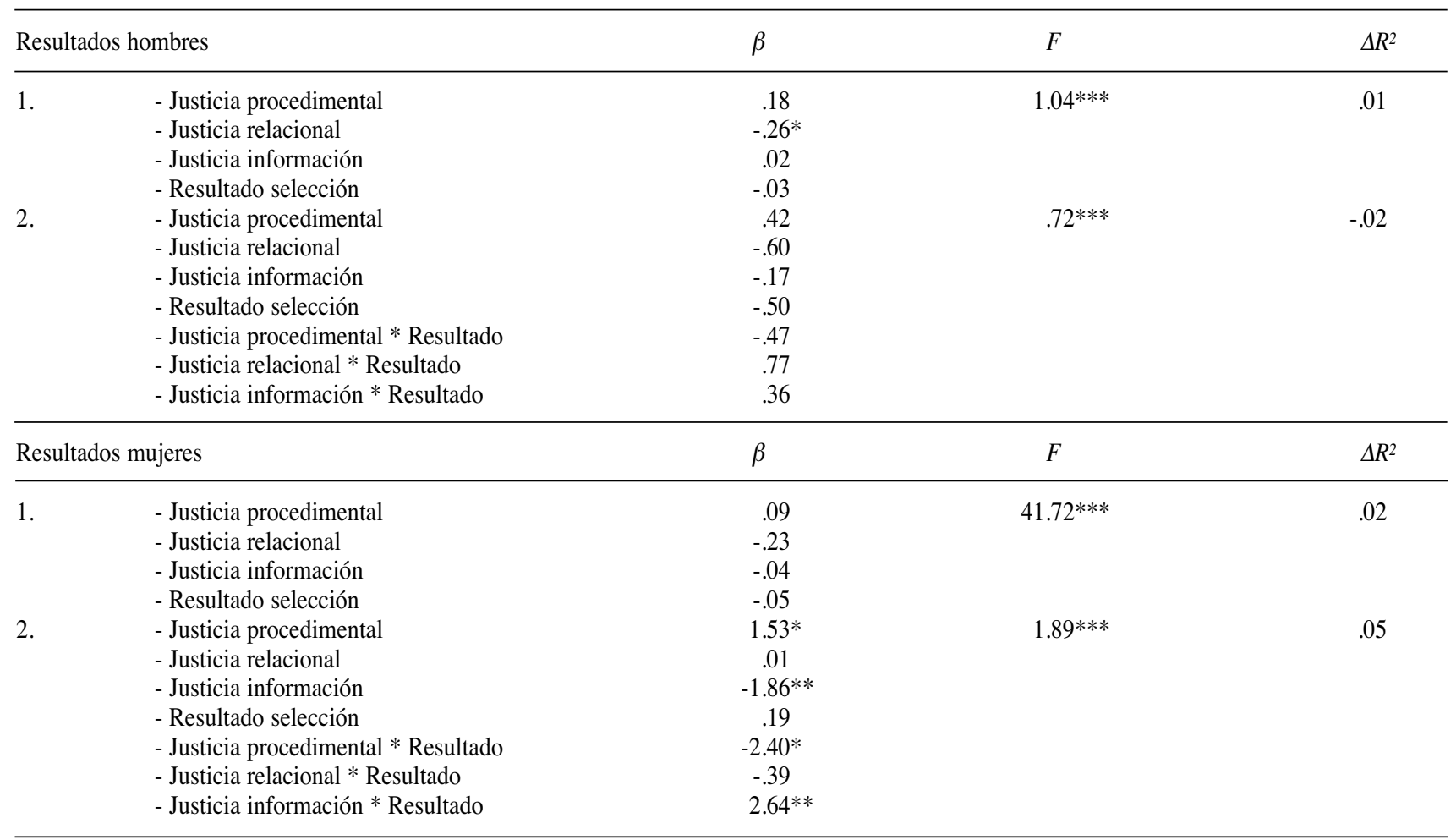

Nota. Resultado en el proceso de SP: 1 No seleccionado y 2 seleccionado.

$\dagger<.10 * \mathrm{p}<.05 * *_{\mathrm{p}}<.01 \quad * * * \mathrm{p}<.001$

iniciar acciones legales se relaciona tanto en hombres como en mujeres con la falta de justicia interaccional, con porcentajes de varianza explicada bajos, pero a tener en cuenta. Como conclusión, y en contra de lo que podría esperarse por las diferencias en los roles de género, a las mujeres durante la selección les influyen mucho los procedimientos utilizados y a los hombres los aspectos más relacionales y de interacción. No obstante, cuando se analiza la posibilidad de iniciar litigios, tanto a hombres como a mujeres les afectan, sobre todo, los aspectos de carácter más personal y afectivo. En este sentido habría que profundizar en qué otras variables, además del género o relacionadas con éste, pueden estar influyendo en estos resultados (tipo de trabajo al que se presentan, etc.).

Por último, no se encuentra que el resultado de la selección actúe modulando el peso de la justicia sobre las intenciones, pues el efecto de la modulación no aumenta la varianza explicada por la influencia directa de la justicia y de los resultados de la selección. Estos resultados coinciden con los de Bauer et al. (2001) quienes encuentran que las percepciones de justicia predicen las intenciones de recomendar incluso después de controlar los resultados de la selección, aunque difiere de otros estudios en los que el resultado es fundamental para explicar las intenciones (p.ej. Brockner y Wiesenfeld, 2005; Maertz et al., 2004). Nuestros resultados enfatizan la importancia de la justicia algo que puede estar relacionado con aspectos como el nivel formativo o la situación laboral de la muestra (un elevado porcentaje acude a la selección para mejorar su situación profesional). Es probable que en estas circunstancias el peso del resultado de la selección se reduzca en favor de otras variables.

Entre las limitaciones del estudio se debe señalar, como ya se ha puesto de manifiesto, que las relaciones entre las intenciones y las conductas son más reducidas de lo que podría esperarse (p. ej. Jones y Skarlicki, 2003). Además siguiendo el planteamiento de Ployhart, Ryan y Bennett (1999) sería interesante examinar cómo se da la información sobre los resultados, ya que parece una variable muy relevante. También sería interesante realizar estudios longitudinales en línea con el trabajo de Smither y cols. (1993), Maertz y cols. (2004) o Anderson y Gotsi (2006), evaluando si la influencia sobre las intenciones se mantiene y durante cuánto tiempo. Por último, sería adecuado utilizar medidas más complejas de las intenciones dado que, en este caso, se han utilizado variables monoítem. Aun así creemos que nuestros resultados ponen de manifiesto la importancia de considerar las percepciones de los candidatos por el efecto en sus intenciones, algo fundamental tanto desde la óptica del Marketing como de la Psicología de las Organizaciones.

Desde una perspectiva aplicada, conocer lo que piensan los candidatos sobre la justicia en selección 
puede permitir elaborar recomendaciones que mejoren los procesos de reclutamiento y la imagen que la organización ofrece a los candidatos, pero también a posibles clientes. Como se ha señalado, la selección supone en muchos casos el primer o, incluso el único contacto con la organización que hace la oferta y, por los datos de este trabajo, habría que cuidar al máximo todo lo que ocurre, tanto los métodos utilizados como las relaciones con los responsables. Además, y muy importante, habría que atender a las diferencias de género, pues apuntan información muy relevante.
Aunque el interés por este tema surge en momentos de pleno empleo, en la actualidad, la crisis económica no debe hacer que las empresas lo descuiden, dada su influencia en la imagen que dan al exterior, en la compra de sus productos, o en el inicio de acciones legales. Así, los resultados de este estudio deberían alertar a las organizaciones de los posibles efectos negativos de determinadas prácticas de selección, y sobre todo de los costes tanto directos como indirectos, que pueden ocasionar procesos que no sean percibidos como justos por los posibles candidatos.

\section{Extended Summary}

Since the 70s in the US and UK, companies and organizations have been taking care of their recruitment and selection procedures to have an argument with which to defend themselves against possible lawsuits for failing to meet anti-discrimination legislation. This has led to develop structured assessment systems focused on test content and on the consistency to manage them (Harris, 2000). In our country there is no tradition in this regard, however, the recent Organic Law 3/2007 for Effective Equality between Women and Men provides a framework for those people who feeling discriminated can exercise their rights. From this perspective, this article has a double objective. First, to study how perceptions of justice in selection process affects on the intentions of the candidates regarding the hiring organization (recommend it to other candidates, to purchase its products or to pursue legal action) and second to identify the influence of the selection outcome (to be or not to be selected). Moreover we will conduct separated analysis for men and women to tests the differences.

In the 90s, Gilliland (1993) and Smither, Reilly, Millsap and Pearlman (1993) suggested that the selection procedures, which are not considered reasonable and not related to the job, generate among the candidates rejection and negative attitudes towards the organization. Saks, Leck and Saunders (1995) showed in a group of students that non-discriminatory surveys produce more positive responses on organizational attractiveness, to job acceptance intentions, and intentions to recommend the hiring organization. Can find results in this address on real candidates (Smith et al., 1995, Wallace, Page \& Lippstreu, 2006). Regarding the justice of tests, Gilliland and Chan (2001) consider that the lack of justice will influence on the intentions towards the organization (Goldman, 2001), about the organizational attractiveness and intentions towards it (Bauer, Maertz, Dolen \& Campion, 1998, Bauer, Truxillo, Sanchez, Craig, Ferrara, \& Campion, 2001, Holtz, Ployhart \& Dominguez, 2005).
Hypothesis 1: We expect relationships between perceptions of fairness in selection and intentions of candidates on the organization making the offer so that those who perceive more justice will show more favourable intentions.

However, other main aspect that influences on selection process perceptions is the outcome (being or not being selected) (e.g. Chan, Schmitt, Sacco \& Deshon, 1998, Elkins \& Phillips, 2000; Hausknecht, Day \& Thomas, 2004, Rolland \& Steiner, 2007). However, interactions have been found between the perception of justice and the result of selection so that fair processes are related with more favourable intentions, especially in those candidates with more favourable results (Ployhart \& Ryan, 1997).

Hypothesis 2: We expect interactions, between perceptions of justice in selection process and the result of selection, on intentions towards the organization.

Regarding the differences of gender, some studies show that men and women respond differently to perceptions of justice (Anderson \& Shinew, 2003) but no important differences are found when evaluating justice in the selection processes (Osca \& GarciaSalmon, 2004; Viswesvaran \& Ones, 2004). In this sense, it is likely that gender does not exert a direct influence but may act through interaction with third variables.

Hypothesis 3: We do not expect significant differences in the perception of justice in selection process between men and women.

Hypothesis 4: We expect differences in how men and women react to what happened during the selection process, so that the dimensions of justice that will influence on intentions will be different for men and women. 


\section{Method}

\section{Procedure and sample}

We collected data from candidates who were participating in selection processes in different geographical areas of Spain. The only condition was that they should have recently participated in a selection process that included an interview. The sample was comprised by 261 candidates, $50.2 \%$ were men (131). The average age was 30.89 years $($ S.D. $=8.5)$ for men and 29.44 (S.D. $=6.5$ ) for women. In the selection process were selected a $72.3 \%$ of women and a $64.3 \%$ of men.

\section{Instruments}

The selection justice was measured with three subscales of the Organizational Justice Scale by Colquitt (2001) but with reference to personnel selection situations: justice on the procedures or procedural justice (9 items), justice in relationships or interactional justice (4 items) and justice in information or informational justice (5 items). Likert response scale were 1 "strongly disagree" and 7 "strongly agree". To assess the intentions regarding the organization we followed the proposal of Macan, Avedon, Paese and Smith (1994) and Smither et al. (1993) and we designed three items enquiring about: intention to recommend the organization to other candidates, intention to purchase its products and intention to pursue legal action. Likert response scale were 1 "very low" and 7 "very high" (Table 1).

\section{Results}

The results of the analysis of variance to prove the hypothesis 1 (Table 2) show, in line with our expectations that the selected candidates have significantly higher scores on three subscales of justice: procedural justice $(F=105.23, p<0.001)$, relational justice $(F=$ $70.15, p<0.001)$ and informational justice ( $F=61.96$, $p<0.001)$. Also, those candidates who have been selected have higher intentions to recommend the organization to other candidates $(F=71.18, p<0.001)$ and to purchase its products $(F=20.59, p<0.001)$ than those not selected, though do not found significant differences in intention to pursue legal action $(F=1.68, p$ $>0.05$ ). Confirming our hypothesis, not significant differences are found between men and women in any of the assessed dimensions of justice: procedural justice $(F=.05, p>0.05)$, relational justice $(F=.42, p>0.05)$ and informational justice $(F=.62, p>0.05)$ (Table 3$)$.

To contrast hypotheses 3 concerning the consequences of justice and the outcome of selection, on the intentions of candidates three regression equations have been carried out (Table 4) and different analysis for men and women have been conducted. In men, the intention to recommend is predicted by interactional justice $(\beta=.21 ; p<0.05)$ and by the outcome of selection $(\beta=.27 ; p<0.001)$, and in women only by procedural justice $(\beta=.37 ; p<0.001)$, with high percentage of explained variance ( $42 \%$ and $43 \%$ respectively). Regarding the intention of men to purchase the organizational products (Table 5), the most predictive variable is informational justice $(\beta=.38 ; p<0.01)$, and in the case of women the procedural justice $(\beta=.38$; $p<0.01)$. Finally, and regarding the intention to pursue legal action (Table 6 ) for men the only significant variable is interactional justice $(\beta=-.26 ; p<0.05)$, and for women procedural justice $(\beta=1.53 ; p<0.05)$ and informational justice $(\beta=-1.86 ; p<0.01)$.

\section{Main Conclusions}

The study takes a step forward on previous work in two aspects, because a sample of real applicants in selection processes was used, and also because as well as procedural justice two other dimensions of justice are used: relational and informative justice. As expected, it was observed that the three dimensions of justice evaluated are related, in the expected direction, with the intention to recommend the hiring organization to others and with the intention to purchase its products, although there are interesting differences in different aspects. Regarding the intention to pursue legal action neither men nor women evaluated have considered this possibility, probably because in our country there is no tradition of legal action due to unfair or discriminatory procedures in personnel selection. This may be the main reason for the absence of clear relationships or with high predictive power. Also, according with our hypothesis, the results of the selection (being selected or unselected), influence in perceptions of justice and in intentions to recommend the organization to others and to purchase its products. However, when perceptions of justice are introduced in the analysis together with the outcomes of selection, the value of outcomes disappears, contrary to the results of other studies (Bauer et al., 1998; Maertz et al., 2004).

Also in line with ours expectations (Osca \& GarciaSalmon, 2004; Viswesvaran \& Ones, 2004), we do not find differences in the perceptions that men and women have on justice in selection processes. However it is confirmed that they are affected by different aspects of justice. In conclusion, and contrary to would be expected in line with differences in social gender roles, during selection process women are greatly influenced by the procedures used and men by more relational and interactional aspects. However, when analyzing the possibility of starting legal actions, both men and women are affected by personal and emotional aspects. In this respect, in the future we should study what other variables may be influencing 
(type of work, "job gender", etc.). Finally, it is not found that the result of selection acts modulating the importance of justice selection on the intentions of candidates, agreeing with Bauer et al. (2001). Finally, further research is needed to address the methodological limitations of our study and to extend our understanding of others moderators in justice of personnel selection. Our results emphasize the need of studying this issue for its impact on very relevant outcomes for organizations.

\section{Referencias}

Anderson, D.M. y Shinew, K.J. (2003). Gender equity in the context of organizational justice: A closer look at a reoccurring issue in the field. Journal of Leisure Research, 35, 228-247.

Anderson, N. y Goltsi, V. (2006). Negative Psychological Effects of Selection Methods: Construct Formulation and an Empirical Investigation into an Assessment Center. International Journal of Selection and Assessment, 14, 236-255.

Bauer, T.N., Maertz, C.P., Dolen, M.R. y Campion, M.A. (1998). Longitudinal assessment of applicant reactions to employment testing and test outcome feedback. Journal of Applied Psychology, 83, 892-903.

Bauer, T.N., Truxillo, D.M., Sánchez, R.J., Craig, J.M., Ferrara, P. y Campion, M.A. (2001). Applicant reactions to selection: Development of the selection procedural justice scale (SPJS). Personnel Psychology, 54, 388-420.

Bernerth, J.B. (2005). Perceptions of Justice in Employment Selection Decisions: The Role of Applicant Gender. International Journal of Selection and Assessment, 13, 206-212.

Brockner, J. y Wiesenfeld, B. (2005). How, when, and why does outcome favorability interact with procedural fairness? En J. Greenberg y J. Colquitt (Eds.), Handbook of organizational justice (pp. 525-554). Mahwah, NJ: Erlbaum.

Chan, D., Schmitt, N., Sacco, J.M. y Deshon, R.P. (1998). Understanding pretest and posttest reactions to cognitive ability and personality tests. Journal of Applied Psychology, 83, 471-485.

Cohen, J. y Cohen, P. (1983). Applied multiple regression/correlation analysis for the behavioural sciences (2nd ed.). Hillsdale, NJ: Erlbaum.

Colquitt, J.A. (2001). On the dimensionality of organizational justice: A construct validation of a measure. Journal of Applied Psychology, 86, 386-400.

Cropanzano, R. y Greenberg, J. (1997). Progress in organizational justice: Tunneling through the maze. En I.T. Robertson y C.L. Cooper (Eds.), International Review of Industrial and Organizational Psychology (Vol. 12, pp. 317-372). New York: Wiley.

Eagly, A.H. y Karau, S.J. (2002). Role congruity theory of prejudice toward female leaders. Psychological Review, 109, 573-598.

Elkins, T.J. y Phillips, J.S. (2000). Job context, selection decision outcome, and the perceived fairness of selection tests: Biodata as an illustrative case. Journal of Applied Psychology, 85, 479-484.

Gilliland, S.W. (1993). The perceived fairness of selection systems: An organizational justice perspective. Academy of Management Review, 18, 694-734.

Gilliland, S.W. (1994). Effects of procedural and distributive justice on reactions to a selection system. Journal of Applied Psychology, 79, 691-701.

Gilliland, S.W. y Chan, D. (2001). Justice in organizations: Theory, methods, and applications. En N. Anderson, D.S. Ones, H.K. Sinangil y C. Viswesvaran (Eds.) Handbook of industrial, work and organizational psychology (pp. 143-165). Thousand Oaks, CA: Sage.

Goldman, B.M. (2001). Toward an understanding of employment discrimination claiming: An integration of organizational justice and social information processing. Personnel Psychology, 54, 361-387.

Harris, L. (2000). Procedural justice and perceptions of fairness in selection practice. International Journal of Selection and Assessment, 8, 148-157.

Hausknecht, J.P., Day, D.V. y Thomas, S.C. (2004). Applicant reactions to selection procedures: An updated model and meta-analysis. Personnel Psychology, 57, 639-683.

Holtz, B.C., Ployhart, R.E. y Domínguez, A. (2005). Testing the Rules of Justice: The Effects of Frame-of-Reference and Pre-Test Validity Information on Personality Test Responses and Test Perceptions. International Journal of Selection and Assessment, 13, 75-86.

Instituto de la Mujer (2008). Encuesta de Población Activa (INE): Tasas de Actividad, Ocupación y Paro. Disponible en: http://www.migualdad.es/mujer/mujeres/cifras/tablas/ Wtrabajo2.XLS

Jones, D.A. y Skarlicki, D.P. (2003). The relationship between perceptions of fairness and voluntary turnover among retail employees. Journal of Applied Social Psychology, 33, 1226-1243.

Landsbergis, P.A., Schnall, P.L., Warren, K., Pickering, T.G. y Schwartz, J.E. (1994). Associations between ambulatory blood pressure and alternative formulations of job strain. Scandinavian Journal of Work, Environment and Health, 20, 349-365.

Ley Orgánica 3/2007, de 22 de marzo, para la Igualdad Efectiva de Mujeres y Hombres. (2007). BOE $n^{\circ} 71$, de 23 de marzo de 2007. Disponible en: http://www.migualdad.es/mujer/politicas/Ley_Igualdad.pdf

Macan, T.H., Avedon, M.J., Paese, M. y Smith, D.E. (1994). The effects of applicants' reactions to cognitive ability tests and an assessment center. Personnel.Psychology, 47, 715-738.

Maertz, C.P., Bauer, T.N., Mosley, D.C., Posthuma, R.A. y Campion, M.A. (2004). Do Procedural Justice Perceptions in a Selection Testing Context Predict Applicant Attraction and Intention Toward the Organization?. Journal of Applied Social Psychology, 34, 125-145.

Moliner, C., Martínez-Tur, V., Peiró, J.M. y Ramos, J. (2005). Linking Organizational Justice to Burnout: Are Men and Women Different? Psychological Reports, 96, 805-816. 
Osca, A. y García Salmones, L. (2004). ¿Cómo perciben los candidatos los procesos de selección? Una aproximación desde el modelo de Justicia Procedimental de Gilliland (1993). Revista de Psicología del Trabajo y de las Organizaciones, 20, 225-248.

Ployhart, R.E. y Ryan, A.M. (1997). Toward and explanation of applicant reactions: An examination of organizational justice and attribution frameworks. Organizational Behavior and Human Decision Processes, 72, 308-335.

Ployhart, R.E., Ryan, A.M. y Bennett, M. (1999). Explanations for selection decisions: Applicants' reactions to informational and sensitivity features of explanations. Journal of Applied Psychology, 84, 87-106.

Rolland, F. y Steiner, D.D. (2007). Test-taker reactions to the selection process: Effects of outcome favorability, explanations, and voice on fairness perceptions. Journal of Applied Social Psychology, 37, 2800-2826.

Rosener, J.B. (1990). Ways women lead. Harvard Business Review, 119-125.

Saks, A.M., Leck, J.D. y Saunders, D.M. (1995). Effects of application blanks and employment equity on applicant reactions and job pursuit intentions. Journal of
Organizational Behavior, 16, 415-430.

Smither, J.W., Reilly, R.R., Millsap, R.E. y Pearlman, K. (1993). Applicant reactions to selection procedures. Personnel Psychology, 46, 49-76.

Steiner, D.D. y Gilliland, S.W. (2001). Procedural Justice in personnel selection: International and cross-cultural perspectives. International Journal of Selection and Assessment, 9, 1-14.

Sweeney, P.D. y McFarlin, D.B. (1997). Process and outcome: Gender differences in the assessment of justice. Journal of Organizational Behavior, 18, 83-98.

Viswesvaran, CH. y Ones, D.S. (2004). Importance of Perceived Personnel Selection System Fairness Determinants: Relations with Demographic, Personality, and Job Characteristics. International Journal of Selection and Assessment, 12, 172-186.

Wallace, J.C., Page, E.E. y Lippstreu, M. (2006). Applicant Reactions to Pre-employment Application Blanks: A Legal and Procedural Justice Perspective. Journal of Business and Psychology, 20, 467-488.

Zedeck, S. (1971). Problems with the use of "moderator" variables. Psychological Bulletin, 76, 295-310.

Manuscrito recibido: 25/10/2009

Revision recibida: $11 / 11 / 2009$

Aceptado: 13/11/2009 